\title{
Analysis of key genes reveal lysine demethylase 5B promotes prostate cancer progression
}

\author{
ZHI YANG, JIAN-XIN XU, DENG-PAN FANG and JIAN KE \\ Department of Urology, The Sixth Hospital of Wuhan, Affiliated Hospital of Jianghan University, \\ Wuhan, Hubei 430015, P.R. China
}

Received August 3, 2019; Accepted January 14, 2020

DOI: $10.3892 / \mathrm{ol} .2020 .11923$

\begin{abstract}
Prostate cancer (PCa) is one of the most common types of cancer in males globally. However, the molecular mechanisms underlying $\mathrm{PCa}$ progression remain largely unclear. In the present study, Gene Expression Omnibus (GEO) datasets and datasets from The Cancer Genome Atlas (TCGA) were used to analyze the expression of lysine demethylase 5B (KDM5B) in PCa. Proliferation, cell cycle and migration assays were used to detect the functional roles of KDM5B. It was found KDM5B was upregulated in PCa tissues by analyzing GEO and TCGA datasets. KDM5B knockdown significantly suppressed proliferation and cell cycle progression in PCa cells. In additional, KDM5B knockdown inhibited PCa cell migration. By analyzing a TCGA dataset, KDM5B was found to be upregulated in patients at N1 stage compared with $\mathrm{N} 0$ stage $\mathrm{PCa}$, in patients at $\mathrm{T} 3+\mathrm{T} 4$ stages compared with T2 stage and in patients with Gleason score $\geq 8$ compared with those with score $\leq 7$. Kaplan-Meier analysis revealed that higher expression of KDM5B was associated with shorter biochemical recurrence-free survival and overall survival time in patients with $\mathrm{PCa}$. These results suggest that expression of KDM5B may serve as a biomarker to predict the outcome of PCa.
\end{abstract}

\section{Introduction}

Prostate cancer (PCa) is the second leading cause of male morbidity and mortality in the world (1). In recent years, the incidence rate of PCa has increased dramatically $(1,2)$. In China in 2015, 603,000 men were estimated to be diagnosed

Correspondence to: Dr Jian-Xin Xu, Department of Urology, The Sixth Hospital of Wuhan, Affiliated Hospital of Jianghan University, 168 Hong Kong Road, Jiang'an, Wuhan, Hubei 430015, P.R. China E-mail: drjianxin@sina.cn

Abbreviations: $\mathrm{PCa}$, prostate cancer; siNC, small interfering RNAs against negative control; KDM5B, lysine demethylase 5B

Key words: differentially expressed gene, KDM5B, PCa, protein-protein interaction analysis, biomarkers with PCa and 266,000 men died of it (3). One of the most likely causes is that more reliable and effective biomarkers for early diagnosis of PCa are still lacking. Although a large number of studies revealed several key proteins in PCa progression, including androgen receptor (AR), forkhead box A1 (FOXA1) and speckle-type BTB/POZ protein (SPOP) (4-7), the pathogenesis and etiology of PCa is still not well-understood. Therefore, here is an urgent need to identify novel regulators to understand mechanisms underlying PCa carcinogenesis and to serve as biomarkers.

Histone methylation is tightly controlled by histone methyltransferases and histone demethylases, and it is one of the most important types of chromatin post-translational modifications (8). Emerging studies have revealed that histone methylation plays a significant role in transcriptional regulation, maintenance of genome integrity and epigenetic inheritance (9-13). An imbalance between methylation and demethylation is frequently observed in the pathogenesis of human disorders, including cancer (14-17). Lysine demethylase $5 \mathrm{~B}$ (KDM5B) is a member of the jumonji/AT-rich interaction domain-containing (ARID) family of histone demethylases, and it is also known as JARID1B or PLU-1. KDM5B has been found to be upregulated in squamous cell carcinoma of the head and neck, breast cancer, hepatocellular carcinoma, gastric cancer and glioma (18-24). Previous studies also revealed that KDM5B is upregulated in PCa (25). However, the functional roles of KDM5B in PCa remain largely unknown.

In the present study, the prognostic value of KDM5B in PCa was explored by analyzing 3 independent public datasets. Moreover, experimental validation was performed by investigating the effects of KDM5B on PCa cell proliferation, cell cycle progression and migration. The current study may provide useful information to explore potential candidate biomarkers for the diagnosis of $\mathrm{PCa}$ and for predicting prognosis in patients.

\section{Materials and methods}

Cell culture. LNCaP, PC-3, 22Rv1, DU145, and WPMY-1 cells were purchased from the American Type Culture Collection. The cell lines were cultured in RPMI-1640 medium (Gibco; Thermo Fisher Scientific, Inc.) supplemented with $10 \%$ fetal bovine serum (FBS; Hyclone; GE Healthcare Life Sciences) in an incubator at $37^{\circ} \mathrm{C}$ with $5 \% \mathrm{CO}_{2}$. 
Cell transfection. Short-interfering RNAs (siRNAs) against KDM5B (siKDM5B-546 and siKDM5B-943) and negative control siRNA (siNC) were purchased from Shanghai GenePharma Co., Ltd. The sequences were as follows: siKDM5B-546 sense, 5'-GCAGUUGUUUGCAAGGAU ATT-3' and antisense, 5'-UAUCCUUGCAAACAACUG CTT-3'; siKDM5B-943 sense, 5'-GCAUCAAGCAAGAAC CUAUTT-3' and antisense, 5'-AUAGGUUCUUGCUUGAUG CTT-3'; and siNC sense, 5'-UUCUCCGAACGUGUCACG UTT-3' and antisense, 5'-ACGUGACACGUUCGGAGAATT-3'. Transfection with the siKDM5Bs or siNC was performed using Lipofectamine 3000 transfection reagent (Invitrogen; Thermo Fisher Scientific, Inc.).

$R N A$ extraction and RT-qPCR. Total RNA was extracted from the cells using the Ultrapure RNA kit (CoWin Biosciences). RT was performed using the SuperQuick RT MasterMix (CoWin Biosciences) according to the manufacturer's protocol. RT-qPCR was performed using the AceQ qPCR SYBR Green Master Mix (Vazyme) according to the manufacturer's protocol. The cycling conditions were as follows: Initial denaturation $\left(2 \mathrm{~min}\right.$ at $95^{\circ} \mathrm{C}$ ) followed by 40 cycles of denaturation (10 sec at $\left.95^{\circ} \mathrm{C}\right)$, annealing $\left(30 \mathrm{sec}\right.$ at $\left.59^{\circ} \mathrm{C}\right)$, elongation $\left(30 \mathrm{sec}\right.$ at $\left.72^{\circ} \mathrm{C}\right)$ and a final extension $\left(30 \mathrm{sec}\right.$ at $\left.72^{\circ} \mathrm{C}\right)$. The PCR primers for mature KDM5B and $\beta$-actin were as follows: KDM5B forward, 5'-AGCAGACTGGCATCTGTAAGG-3' and reverse, 5'-GAA GTTTATCAACATCACATGCAA- 3 '; and $\beta$-actin forward, 5'-CCTCTCCCAAGTCCACACAG-3' and reverse, 5'-GGG CACGAAGGCTCATCATT-3'. $\beta$-actin was used as internal control. The $2^{-\Delta \Delta C q}$ method was used to analyze the data (26). Each sample was measured in triplicate.

Western blot analysis. The PCa cells were homogenized and sonicated using Mammalian Protein Extraction Kit (CoWin Biosciences). Protein concentrations were detected using a BCA Protein Quantification kit, according to the manufacturer's protocol. The proteins $(50 \mu \mathrm{g})$ were separated by $10 \%$ SDS-PAGE and then transferred onto polyvinylidene difluoride membranes. The membrane was blocked with 5\% non-fat dry milk for $1 \mathrm{~h}$ at room temperature and incubated with specific primary antibodies overnight at $4^{\circ} \mathrm{C}$. The primary antibodies used were as follows: KDM5B (1:1,000; Abnova), ACTB (1:1,000, cat. no. ab8226; Abcam). The secondary antibodies were Goat Anti-Mouse IgG H\&L (1:1,000, cat. no. ab205719; Abcam) for ACTB, and Goat Anti-Rabbit IgG H\&L (1:1,000, cat. no. ab205719; Abcam) for KDM5B. The blots were detected with an enhanced chemiluminescence substrate kit (Thermo Fisher Scientific, Inc.), according to the manufacturer's protocol. The bands were scanned and quantified by ImageJ v1.47 software (National Institutes of Health).

Cell proliferation assay. Cell proliferation was detected using the Cell Counting Kit-8 (CCK-8) assay (MedChemExpress) in 96-well plates. After transfection, $100 \mu \mathrm{l} /$ well of cells were added to 96-well plates. CCK-8 reagent was added to each well $2 \mathrm{~h}$ before the end of the experiment, and the cells were incubated, and the absorbance was then measured at $450 \mathrm{~nm}$ wavelength in a microplate reader.

Cell cycle analysis. Transfected $\mathrm{LNCaP}$ and PC-3 cells were collected $48 \mathrm{~h}$ post-transfection. Triton X-100 (0.03\%) and propidium iodide $(50 \mathrm{ng} / \mathrm{ml})$ were used to resuspend cells. After incubation at room temperature for $10 \mathrm{~min}$, the transfected cells were examined using a flow cytometer (Beckman Coulter, Inc.). Each sample was measured in triplicate.

Cell migration assay. Cells were treated with siRNAs, including siNC, siKDM5B-546 and siKDM5B-943. Transwell plates were used for the determination of migration ability. Briefly, $700 \mu 11640$ medium supplemented with 10\% FBS was added to the lower chamber of the Transwells. A total of 2,000 cells were resuspended in $100 \mu 11640$ medium supplemented with $1 \%$ FBS, and then added to the upper chamber of the system. After incubation in an incubator at $37^{\circ} \mathrm{C}$ for 3 days, the chambers were removed and unmigrated cells in the upper chamber were wiped with a cotton swab. The migrated cells in the upper chamber were washed twice with PBS and were then fixed with $700 \mu 1$ methanol for $15 \mathrm{~min}$. Next, the migrated cells were stained with DAPI for 20 min, then washed 3 times with PBS. Cells were imaged with a microscope. Each sample was measured in triplicate.

Statistical analysis. The data are presented as the mean \pm SD. Student's t-test or Mann-Whitney U-test were used to compare the difference between the 2 groups of data. Correlation analysis was performed with Spearman's rank correlation. For multiple groups, Statistical analyses were performed using a one-way analysis of variance with the Bonferroni test for post hoc comparisons. Survival analysis was based on the Kaplan-Meier method and the log-rank tests to compare the differences between survival curves. $\mathrm{P}<0.05$ was considered to indicate a statistically significant difference.

\section{Results}

KDM5B is upregulated in PCa. The expression levels of KDM5B in PCa and normal samples were previously unknown. In the present study, 3 independent datasets were analyzed, including TCGA, GSE17951 (27) and the Prensner datasets (28). KDM5B was found to be significantly upregulated in PCa tissues by analyzing TCGA (Fig. 1A). To further validate this result, two additional independent datasets, GSE17951 and Prensner were analyzed, and consistent results were observed (Fig. 1B and C). To further compare KDM5B protein levels in $\mathrm{PCa}$ and normal tissues, KDM5B protein expression was analyzed using the Human Protein Atlas. KDM5B protein was upregulated in PCa samples, however, KDM5B was not detected in normal prostate glandular cells (Fig. 1E). KDM5B expression was also detected in cell lines, including LNCaP, PC-3, DU145, and 22Rv1 PCa cells and WPMY-1 noncancerous prostate cells. KDM5B was found to be upregulated in PCa cell lines compared to WPMY-1 cells (Fig. 1D).

The knockdown of KDM5B inhibits PCa cell proliferation. The roles of KDM5B on the proliferation of PCa cells was then evaluated. siRNAs against KDM5B (siKDM5B-546 and siKDM5B-943) were designed to knockdown KDM5B expression. LNCaP and PC-3 cells were transfected with siNC or siKDM5B siRNAs. At $48 \mathrm{~h}$ post-transfection, both the mRNA and protein levels of KDM5B were significantly suppressed in both siKDM5B groups compared with the 

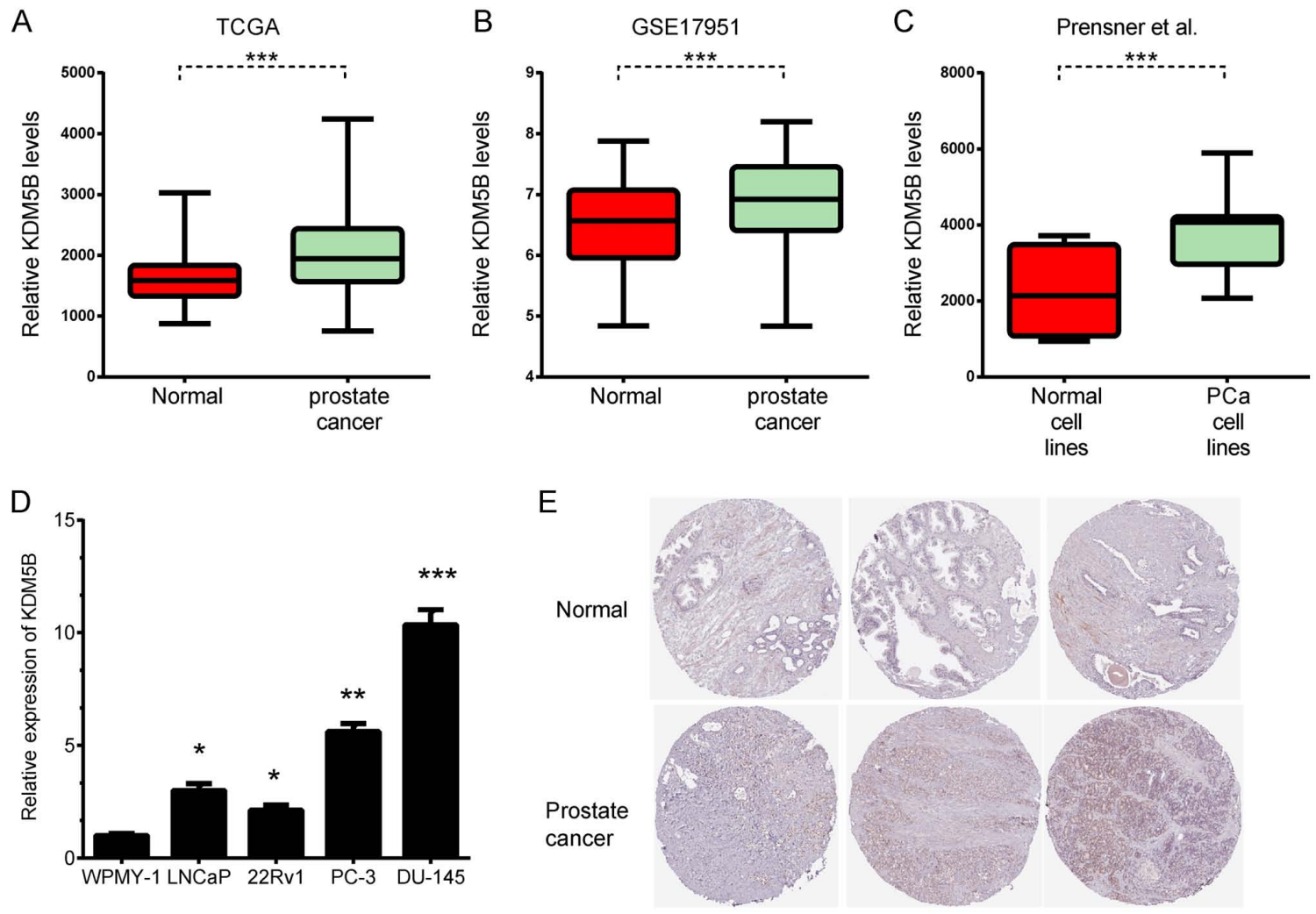

Figure 1. KDM5B is upregulated in prostate cancer. KDM5B is upregulated in (A) TCGA, (B) GSE17951 and (C) Prensner datasets. ${ }^{* * *} \mathrm{P}<0.001$. (D) Expression of KDM5B mRNA in WPMY-1, LNCaP, 22RV1, DU145 and PC-3 cells. (E) Protein levels of KDM5B in normal prostate and prostate cancer samples by analyzing the Human Protein Atlas. ${ }^{*} \mathrm{P}<0.05,{ }^{* *} \mathrm{P}<0.01,{ }^{* * *} \mathrm{P}<0.001$ vs. WPMY-1. KDM5B, lysine demethylase 5B; TCGA, The Cancer Genome Atlas.
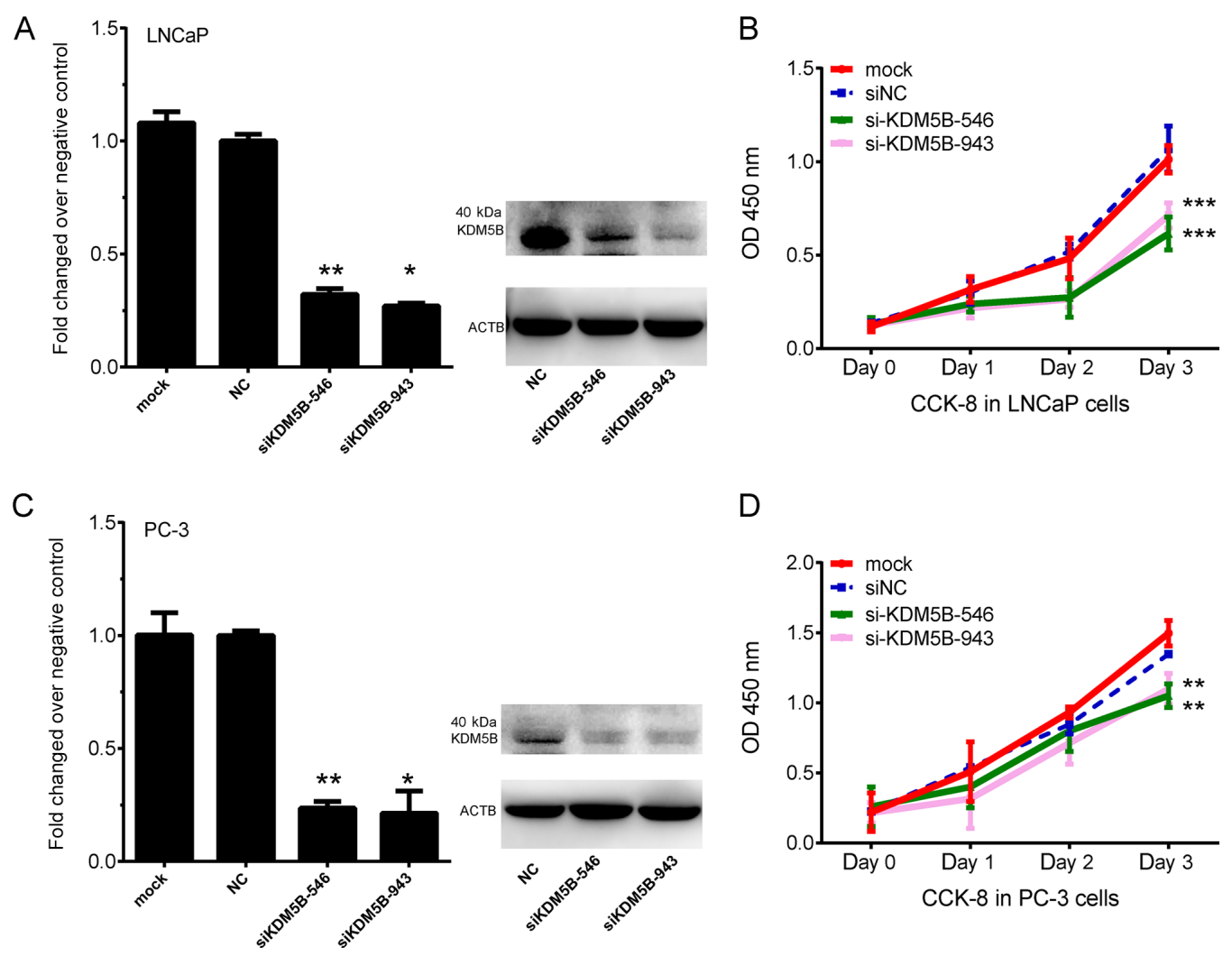

Figure 2. Knockdown of KDM5B inhibits PCa cell proliferation. (A) mRNA and protein expression of KDM5B after transfection with mock, siNC, siKDM5B-546 and siKDM5B-943 in LNCaP cells. (B) Knockdown of KDM5B inhibits LNCaP cell proliferation. (C) mRNA and protein expression of KDM5B after transfection with mock, siNC, siKDM5B-546 and siKDM5B-943 in PC-3 cells. (D) Knockdown of KDM5B inhibits PC-3 cell proliferation. ${ }^{*} \mathrm{P}<0.05,{ }^{* *} \mathrm{P}<0.01,{ }^{* * * *} \mathrm{P}<0.001$ vs. NC. KDM5B, lysine demethylase 5B; PCa, prostate cancer; si, small interfering; NC, negative control; OD, optical density; CCK-8, Cell Counting Kit-8. 
A

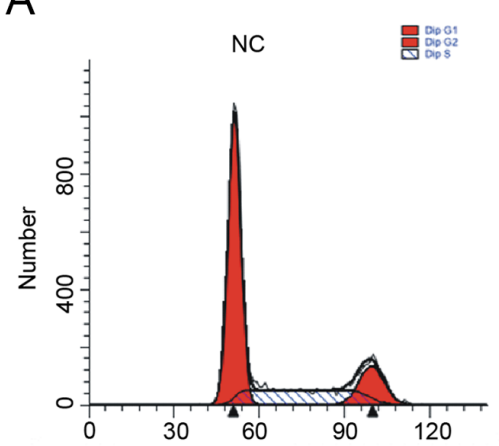

$\mathrm{B}$

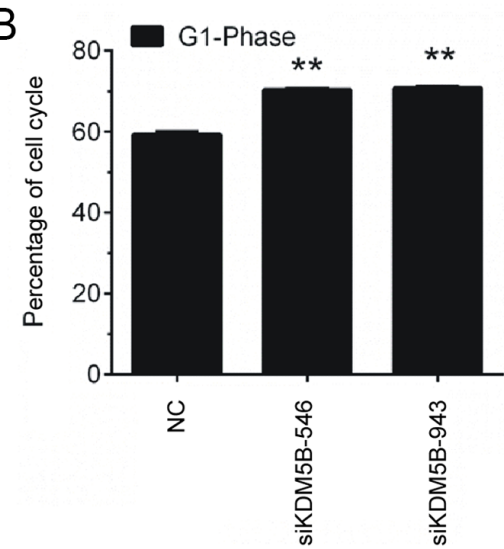

C
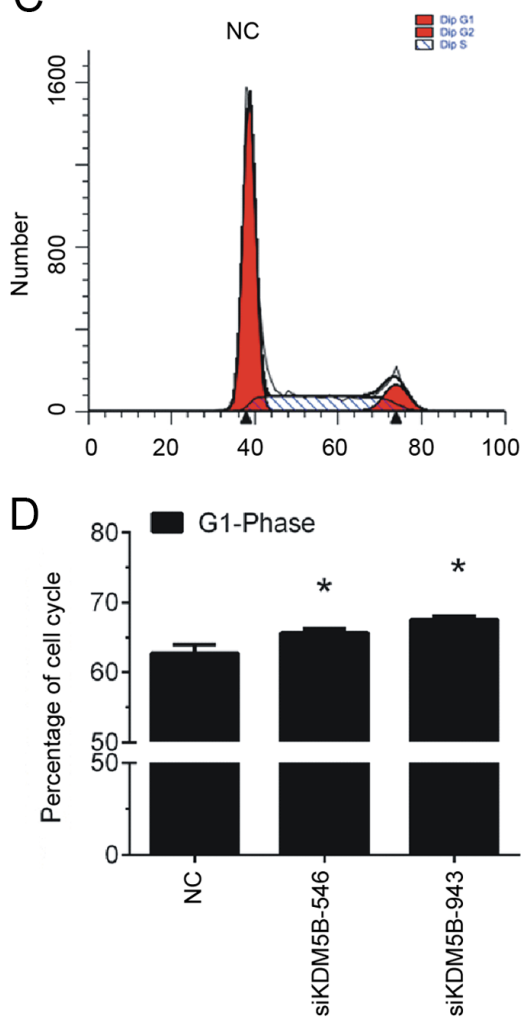

PC-3
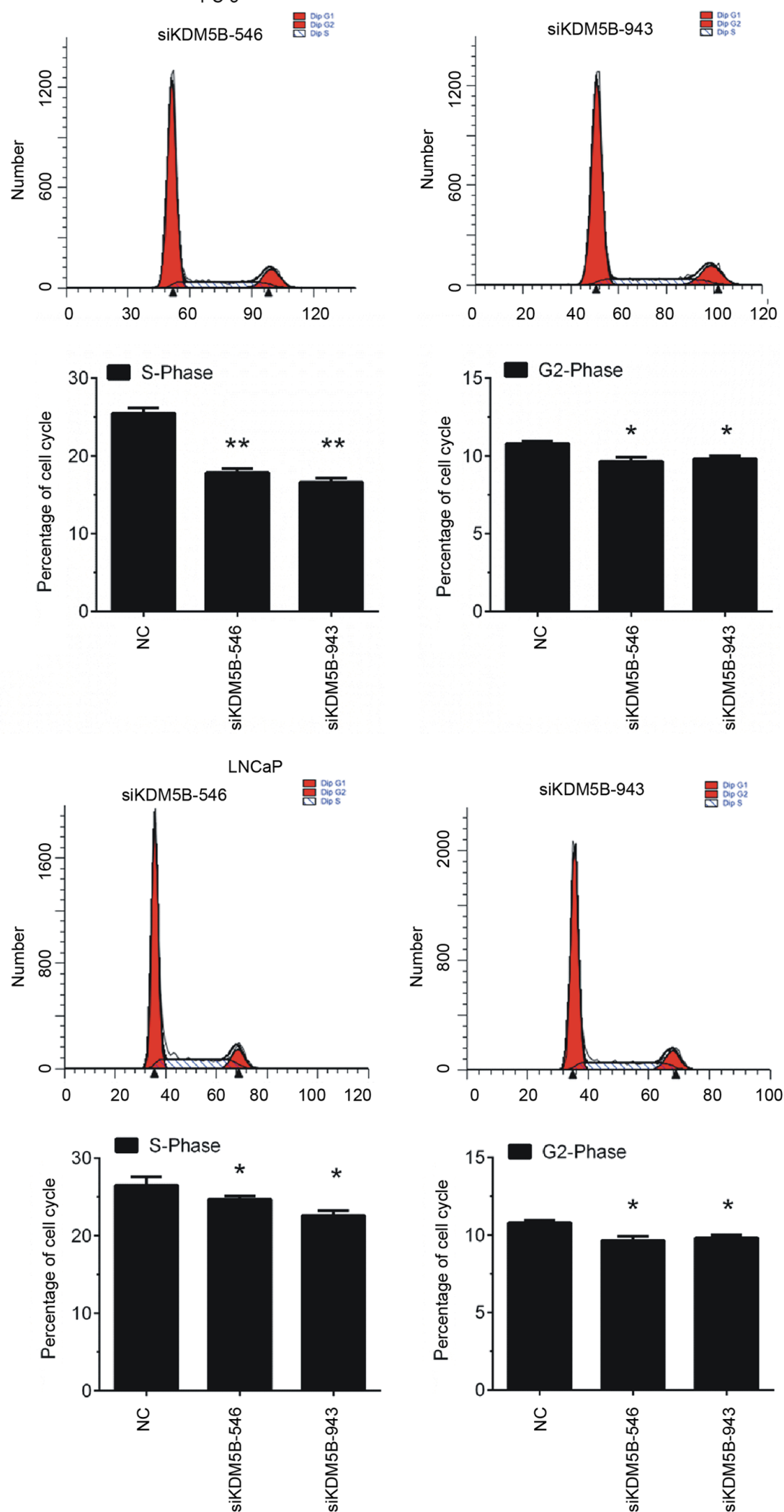
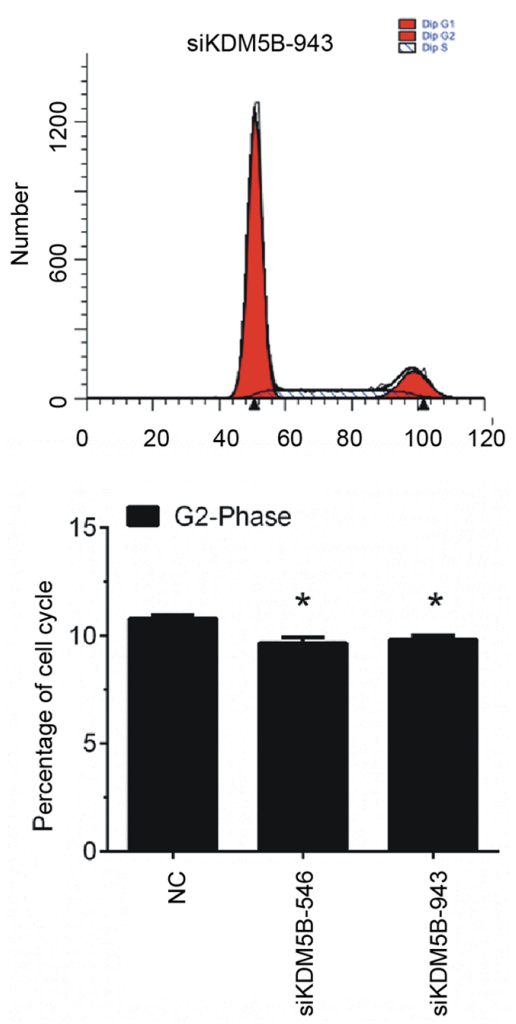

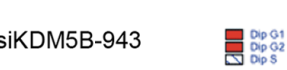

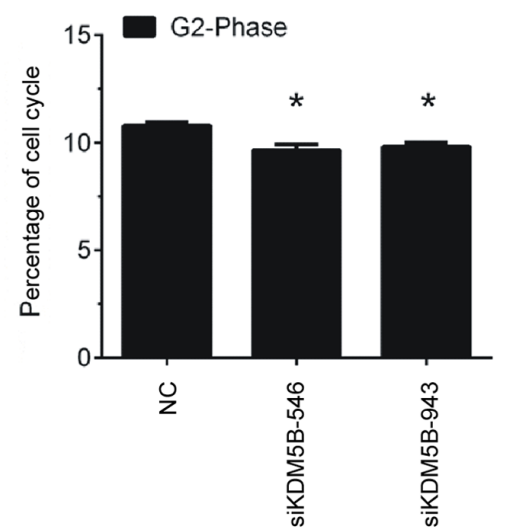

Figure 3. Knockdown of KDM5B induced prostate cancer cell cycle arrest at the $\mathrm{G}_{1}$ phase. (A and B) Knockdown of KDM5B induced PC3 cell cycle arrest at the $\mathrm{G}_{1}$ phase. (C and D) Knockdown of KDM5B induced LNCaP cell cycle arrest at the $\mathrm{G}_{1}$ phase. ${ }^{*} \mathrm{P}<0.05,{ }^{* *} \mathrm{P}<0.01$ vs. NC. KDM5B, lysine demethylase $5 \mathrm{~B}$; si, small interfering; $\mathrm{NC}$, negative control.

siNC group $(\mathrm{P}<0.05$ and $\mathrm{P}<0.01 ;$ Fig. $2 \mathrm{~A}$ and $\mathrm{C})$. Additionally, knockdown of KDM5B inhibited proliferation of LNCaP and PC-3 cells $(\mathrm{P}<0.001$ and $\mathrm{P}<0.001$; Fig. $2 \mathrm{~B}$ and $\mathrm{D})$.
Knockdown of KDM5B induces PCa cell cycle arrest in the G1 phase. Furthermore, the effects of KDM5B on cell cycle progression of $\mathrm{LNCaP}$ and $\mathrm{PC}-3$ cells were detected by flow 
A
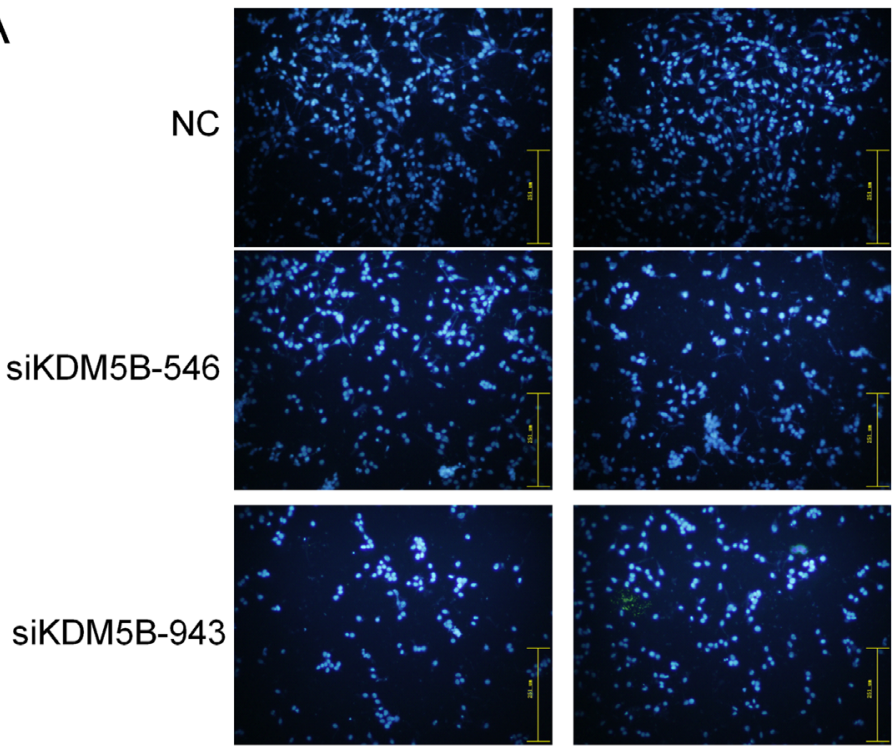

B

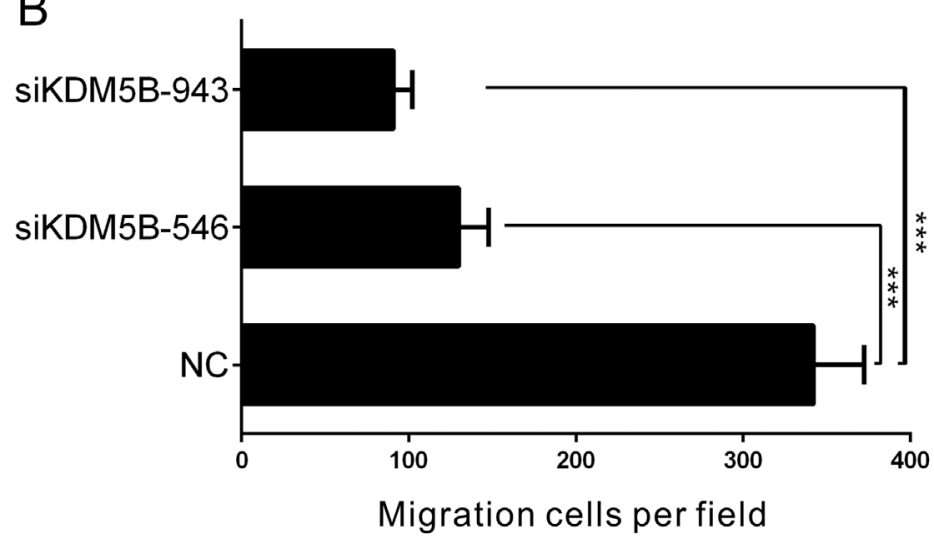

Figure 4. Knockdown of KDM5B inhibits migration of prostate cancer cells in vitro. (A and B) Knockdown of KDM5B inhibited cell migration in PC-3 cells. Each experiment was performed in triplicate. $\mathrm{n}=3$. Bars represent the mean \pm SD. Scale bar, $251 \mu \mathrm{m} .{ }^{* * *} \mathrm{P}<0.001$. KDM5B, lysine demethylase $5 \mathrm{~B}$; si, small interfering; NC, negative control.

cytometry. The findings revealed that knockdown of KDM5B in LNCaP and PC-3 cells induced a significant increase in the proportion of cells in G1 phase, however, KDM5B knockdown decreased the proportion of cells in $\mathrm{S}$ and $\mathrm{G} 2 / \mathrm{M}$ phase $(\mathrm{P}<0.05$ and $\mathrm{P}<0.01$; Fig. 3).

The knockdown of KDM5B inhibits the migration of $\mathrm{PCa}$ cells in vitro. The number of PC-3 cells that migrated through the filter of the Transwell chambers was used to estimate the migratory ability of the cells (Fig. 4). Compared with the scrambled siRNA-treated control group, the number of migrating cells was decreased by 2.74- and 3.91-fold in PC-3 cells treated with siKDM5B-546 and siKDM5B-943, respectively $(\mathrm{P}<0.001$; Fig. 4$)$.

KDM5B expression is associated with clinical variables in patients with PCa. The association between KDM5B expression and the clinicopathological characteristics of patients with PCa was investigated. As presented in Fig. 5, KDM5B expression was significantly upregulated in N1 stage PCa samples compared to N0 samples (Fig. 5A), in T3/T4 PCa samples compared to T2 samples (Fig. 5B). Moreover, we analyzed the correlation between KDM5B expression and Gleason score in patients with $\mathrm{PCa}$. The results showed that the higher expression levels of KDM5B significantly correlated to the higher Gleason score in PCa patients (Fig. 5C-D).

In addition, Kaplan-Meier analysis was performed to determine whether KDM5B expression was associated with biochemical recurrence (BCR)-free survival and overall survival in patients with PCa by analyzing the TCGA dataset. In order to divide all PCa samples into groups based on the high and low expression of KDM5B, the cut-off value was calculated using the Cutoff Finder (http://molpath.charite. de/cutoff/) (29). In TCGA analysis, the BCR-free survival and overall survival rates were higher in the KDM5B-low compared with the KDM5B-high patients (Fig. 5E and F). These results indicate that KDM5B expression may serve as a biomarker to predict the prognosis of patients with PCa.

\section{Discussion}

In recent decades, studies focused on exploring the functions of several key proteins, such as AR, SPOP, and FOXA1 in PCa (4-7), however, the molecular mechanisms underlying PCa progression remain largely unclear. Following the application of high-throughput screening techniques like microarray 

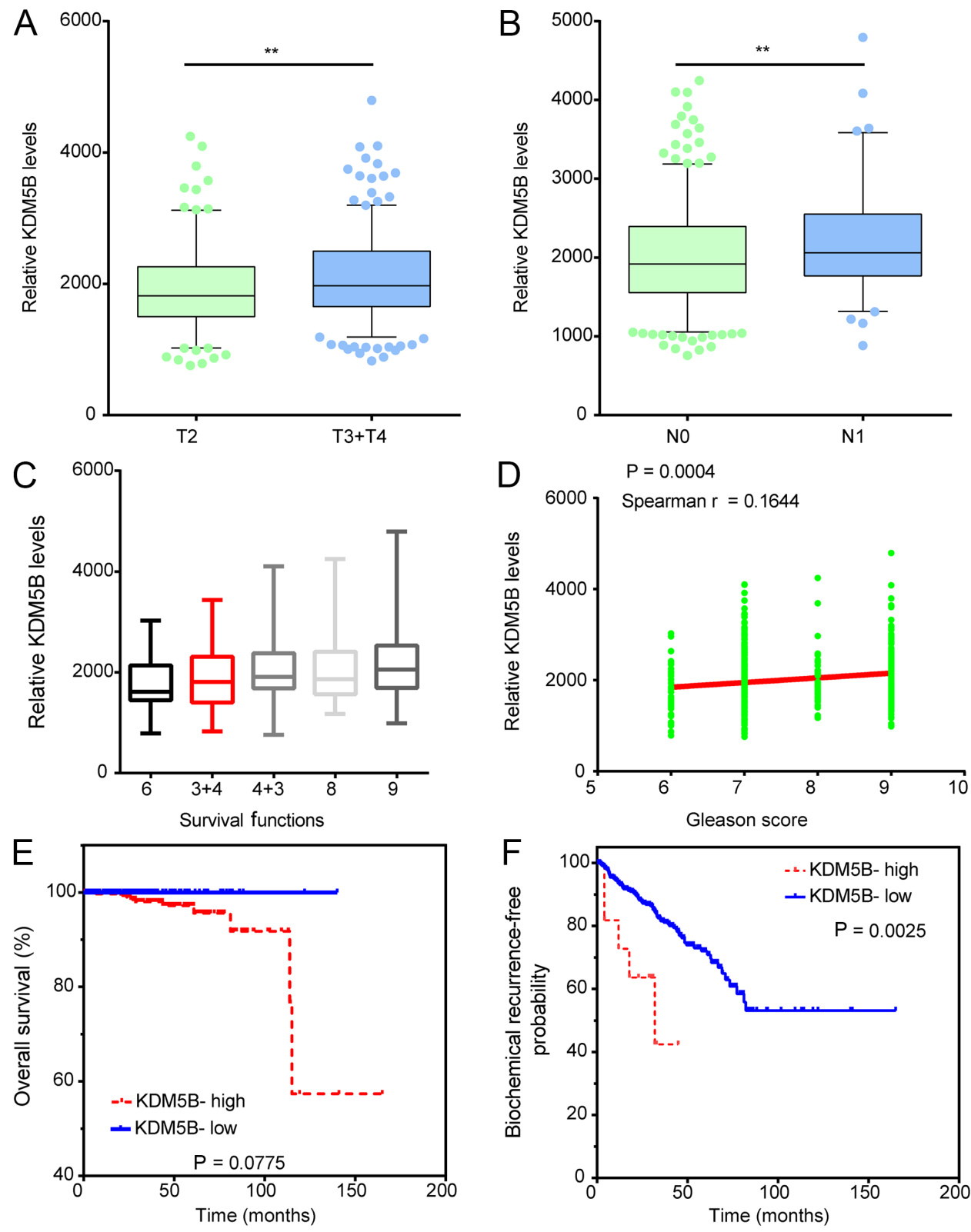

Figure 5. KDM5B expression is associated with PCa clinical variables. (A) KDM5B expression was upregulated in T3/4 prostate cancer compared with T2 prostate cancer. (B) KDM5B expression was upregulated in N1 prostate cancer compared with N0 prostate cancer. (C) KDM5B expression was upregulated in Gleason Score 7, 8 and 9 PCa samples compared with Gleason Score 6 PCa. (D) Higher expression levels of KDM5B significantly correlated to the higher Gleason score in patients with PCa using Spearman's rank correlation analysis. Comparing with patients with low KDM5B expression, the (E) overall survival rates and (F) biochemical recurrence-free survival rates were lower in patients with high KDM5B expression in The Cancer Genome Atlas dataset. Survival analysis was based on the Kaplan-Meier method and the log-rank tests to compare the differences between survival curves. ${ }^{* *} \mathrm{P}<0.01$. KDM5B, lysine demethylase $5 \mathrm{~B} ; \mathrm{PCa}$, prostate cancer.

and small RNA sequencing, a series of studies identified genes associated with PCa progression. For example, a study by Taylor et al (30) and TCGA groups performed integrative genomic analysis of human PCa. In the present study, a comprehensive analysis of $\mathrm{PCa}$-related genes was performed by using 3 public datasets, TCGA and GSE17951, and KDM5B was found to be upregulated in PCa samples.

KDM5B was included in this hub-network. The functional roles of KDM5B in PCa remain largely unknown. Previous studies had observed that dysregulation of KDM5B was associated with cancer progression. KDM5B was found to be upregulated in squamous cell carcinoma of the head and neck, breast cancer, hepatocellular carcinoma, gastric cancer and glioma (18-24). These studies suggested that KDM5B may serve as a diagnostic and therapeutic target for cancers. In the present study, the function of KDM5B was explored in $\mathrm{PCa}$ cells. KDM5B was found to act as an oncogene in PCa cells, as knockdown of KDM5B significantly inhibited cell proliferation, cell cycle progression, and migration. To the best of our knowledge, this is the first study to reveal the effects of KDM5B on the biological functions of PCa cells.

Prostate-specific antigen testing is the most widely used biomarker for patients with PCa, but its efficacy is limited by low specificity. Of note, several recent studies revealed that Low serum total testosterone level and Body mass index could serve as a predictor of upstaging and upgrading in low-risk 
prostate cancer patients. For example, Ferro et al reported that low serum total testosterone levels as a predictor of upstaging and upgrading in low-risk prostate cancer patients meeting the inclusion criteria for active surveillance (31). And de Cobelli et al revealed that Body mass index was associated with upstaging and upgrading in patients with low-risk prostate cancer who met the inclusion criteria for active surveillance (32). Moreover, the expression levels of multiple protein coding genes or non-protein coding genes were also revealed to be associated with the progression and prognosis of patients with PCa, such as PHI, PCA3, sarcosine, and Urotensin II receptor. For example, de Cobelli et al showed Urotensin II receptor on preoperative biopsy is associated with upstaging and upgrading in prostate cancer (33). Sreekumar and his colleges found sarcosine levels in PCa samples were associated with the progression of cancer (34). However, lacking reliable and effective biomarkers for $\mathrm{PCa}$ diagnosis remained to be one of the biggest challenges in $\mathrm{PCa}$ treatment was lacking reliable and effective biomarkers for PCa diagnosis. In the current study, KDM5B was evaluated as a potential biomarker for PCa. By analyzing public datasets, KDM5B was found to be upregulated in $\mathrm{PCa}$ compared with normal samples, in T3/T4 PCa samples compared with T2 samples, in N1 stage samples compared to N0 samples and in Gleason score, $\geq 8$ samples compared to Gleason score $\leq 7$ samples. Moreover, overall survival rates were higher in patients with low expression of KDM5B compared with those with high expression. These results indicate that KDM5B expression may serve as a biomarker of $\mathrm{PCa}$. We also realized that the combined analysis of KDM5B levels and other potential biomarkers, such as low serum total testosterone level, Body mass index, PHI, PCA3, sarcosine, and Urotensin II receptor levels, in PCa samples using clinical samples could strength the clinical importance of KDM5B in PCa.

In conclusion, 3 public datasets were analyzed to identify differentially expressed genes in PCa. A total of 3,834 genes were found to be dysregulated in PCa. Bioinformatic analysis revealed that these DEGs were associated with cell cycle, translation, and metabolic pathways. PPI network analysis revealed that KDM5B was a key regulator in $\mathrm{PCa}$ progression. Knockdown of KDM5B in PCa cells significantly inhibited proliferation, cell cycle progression and migration. In addition, KDM5B was upregulated in PCa tissues and associated with PCa clinical variables. High expression of KDM5B was associated with worse prognosis in patients with PCa. Given these results, KDM5B may be a potential therapeutic target for PCa.

\section{Acknowledgements}

Not applicable.

\section{Funding}

No funding was received.

\section{Availability of data and materials}

All data generated or analyzed during the present study are included in this published article.

\section{Authors' contributions}

Conception and design of the study was conducted by ZY and JXX. Development of methodology was conducted by ZY, JXX, DPF and JK. ZY, JXX, DPF and JK performed the analysis and interpretation of data, and wrote, reviewed and revised the manuscript. All authors read and approved the final manuscript.

\section{Ethics approval and consent to participate}

Not applicable.

\section{Patient consent for publication}

Not applicable.

\section{Competing interests}

The authors declare that they have no competing interests.

\section{References}

1. Siegel RL, Miller KD and Jemal A: Cancer statistics, 2017. CA Cancer J Clin 67: 7-30, 2017.

2. Siegel RL, Miller KD and Jemal A: Cancer statistics, 2016. CA Cancer J Clin 66: 7-30, 2016.

3. Chen W, Zheng R, Baade PD, Zhang S, Zeng H, Bray F, Jemal A, $\mathrm{Yu}$ XQ and He J: Cancer statistics in China, 2015. CA Cancer J Clin 66: 115-132, 2016.

4. Antonarakis ES, Lu C, Wang H, Luber B, Nakazawa M, Roeser JC, Chen Y, Mohammad TA, Chen Y, Fedor HL, et al: AR-V7 and resistance to enzalutamide and abiraterone in prostate cancer. N Engl J Med 371: 1028-1038, 2014.

5. Sahu B, Laakso M, Ovaska K, Mirtti T, Lundin J, Rannikko A, Sankila A, Turunen JP, Lundin M, Konsti J, et al: Dual role of FoxA1 in androgen receptor binding to chromatin, androgen signalling and prostate cancer. EMBO J 30: 3962-3976, 2011.

6. Barbieri CE, Baca SC, Lawrence MS, Demichelis F, Blattner M, Theurillat JP, White TA, Stojanov P, Van Allen E, Stransky N, et al: Exome sequencing identifies recurrent SPOP, FOXA1 and MED12 mutations in prostate cancer. Nat Genet 44: 685-689, 2012.

7. Geng C, Rajapakshe K, Shah SS, Shou J, Eedunuri VK, Foley C, Fiskus W, Rajendran M, Chew SA,Zimmermann M, et al: Androgen receptor is the key transcriptional mediator of the tumor suppressor SPOP in prostate cancer. Cancer Res 74: 5631-5643, 2014.

8. Kouzarides T: Histone methylation in transcriptional control. Curr Opin Genet Dev 12: 198-209, 2002.

9. Berger SL: Histone modifications in transcriptional regulation. Curr Opin Genet Dev 12: 142-148, 2002.

10. Kulis M, Queirós AC, Beekman R and Martín-Subero JI: Intragenic DNA methylation in transcriptional regulation, normal differentiation and cancer. Biochim Biophys Acta 1829: 1161-1174, 2013.

11. Sharif J, Muto M, Takebayashi S, Suetake I, Iwamatsu A, Endo TA, Shinga J, Mizutani-Koseki Y, Toyoda T, Okamura K, et al: The SRA protein Np95 mediates epigenetic inheritance by recruiting Dnmt1 to methylated DNA. Nature 450: 908-912, 2007.

12. Fukuda A and Hisatake K: Histone methylation and transcriptional regulation. Seikagaku 79: 362-365, 2007 (In Japanese).

13. An W: Histone acetylation and methylation: Combinatorial players for transcriptional regulation. Subcell Biochem 41: 351-369, 2007.

14. Schlesinger Y, Straussman R, Keshet I, Farkash S, Hecht M, Zimmerman J,EdenE, YakhiniZ,Ben-ShushanE,ReubinoffBE, et al: Polycomb-mediated methylation on Lys27 of histone $\mathrm{H} 3$ pre-marks genes for de novo methylation in cancer. Nat Genet 39: 232-236, 2007.

15. Kulis $M$ and Esteller M: DNA methylation and cancer. Adv Genet 70: 27-56, 2010.

16. de Sousa E Melo F, Colak S, Buikhuisen J, Koster J, Cameron K, de Jong JH, Tuynman JB, Prasetyanti PR, Fessler E, van den Bergh SP, et al: Methylation of cancer-stem-cell-associated Wnt target genes predicts poor prognosis in colorectal cancer patients. Cell Stem Cell 9: 476-485, 2011. 
17. Parrella P, Poeta ML, Gallo AP, Prencipe M, Scintu M Apicella A, Rossiello R, Liguoro G, Seripa D, Gravina C, et al: Nonrandom distribution of aberrant promoter methylation of cancer-related genes in sporadic breast tumors. Clin Cancer Res 10: 5349-5354, 2004.

18. Hayami S, Yoshimatsu M, Veerakumarasivam A, Unoki M, Iwai Y, Tsunoda T, Field HI, Kelly JD, Neal DE, Yamaue H, et al: Overexpression of the JmjC histone demethylase KDM5B in human carcinogenesis: Involvement in the proliferation of cancer cells through the E2F/RB pathway. Mol Cancer 9: 59, 2010.

19. Barrett A, Santangelo S, Tan K, Catchpole S, Roberts K, Spencer-Dene B, Hall D, Scibetta A, Burchell J, Verdin E, et al: Breast cancer associated transcriptional repressor PLU-1/JARID1B interacts directly with histone deacetylases. Int J Cancer 121: 265-275, 2007.

20. Wang D, Han S, Peng R, Jiao C, Wang X, Yang X, Yang R and Li X: Depletion of histone demethylase KDM5B inhibits cell proliferation of hepatocellular carcinoma by regulation of cell cycle checkpoint proteins p15 and p27. J Exp Clin Cancer Res 35: 37, 2016.

21. Wang Z, Tang F, Qi G, Yuan S, Zhang G, Tang B and He S: KDM5B is overexpressed in gastric cancer and is required for gastric cancer cell proliferation and metastasis. Am J Cancer Res 5: 87-100, 2015.

22. Dai B, Hu Z, Huang H, Zhu G, Xiao Z, Wan W, Zhang P, Jia W and Zhang L: Overexpressed KDM5B is associated with the progression of glioma and promotes glioma cell growth via downregulating p21. Biochem Biophys Res Commun 454: 221-227, 2014.

23. Bamodu OA, Huang WC, Lee WH, Wu A, Wang LS, Hsiao M, Yeh CT and Chao TY: Aberrant KDM5B expression promotes aggressive breast cancer through MALAT1 overexpression and downregulation of hsa-miR-448. BMC Cancer 16: 160, 2016

24. Catchpole S, Spencer-Dene B, Hall D, Santangelo S, Rosewell I, Guenatri M, Beatson R, Scibetta AG, Burchell JM and Taylor-Papadimitriou J: PLU-1/JARID1B/KDM5B is required for embryonic survival and contributes to cell proliferation in the mammary gland and in ER+ breast cancer cells. Int J Oncol 38 : $1267-1277,2011$

25. Xiang Y, Zhu Z, Han G, Ye X, Xu B, Peng Z, Ma Y, Yu Y, Lin H, Chen AP and Chen CD: JARID1B is a histone H3 lysine 4 demethylase up-regulated in prostate cancer. Proc Natl Acad Sci USA 104: 19226-19231, 2007.

26. Livak KJ and Schmittgen TD: Analysis of relative gene expression data using real-time quantitative PCR and the 2(-Delta Delta C(T)) method. Methods 25: 402-408, 2001.
27. Wang Y, Xia XQ, Jia Z, Sawyers A, Yao H, Wang-Rodriquez J, Mercola D and McClelland M: In silico estimates of tissue components in surgical samples based on expression profiling data. Cancer Res 70: 6448-6455, 2010.

28. Prensner JR, Iyer MK, Balbin OA, Dhanasekaran SM, Cao Q, Brenner JC, Laxman B, Asangani IA, Grasso CS, Kominsky HD, et al: Transcriptome sequencing across a prostate cancer cohort identifies PCAT-1, an unannotated lincRNA implicated in disease progression. Nat Biotechnol 29: 742-749, 2011.

29. Budczies J, Klauschen F, Sinn BV, Győrffy B, Schmitt WD, Darb-Esfahani S and Denkert C: Cutoff Finder: A comprehensive and straightforward Web application enabling rapid biomarker cutoff optimization. PLoS One 7: e51862, 2012.

30. Taylor BS, Schultz N, Hieronymus H, Gopalan A, Xiao Y, Carver BS, Arora VK, Kaushik P, Cerami E Reva B, et al: Integrative genomic profiling of human prostate cancer. Cancer Cell 18: 11-22, 2010.

31. Ferro M, Lucarelli G, Bruzzese D, Di Lorenzo G, Perdonà S, Autorino R, Cantiello F, La Rocca R, Busetto GM, Cimmino A, et al: Low serum total testosterone level as a predictor of upstaging and upgrading in low-risk prostate cancer patients meeting the inclusion criteria for active surveillance. Oncotarget 8: 18424-18434, 2017.

32. de Cobelli O, Terracciano D, Tagliabue E, Raimondi S, Galasso G, Cioffi A, Cordima G, Musi G, Damiano R, Cantiello F, et al: Body mass index was associated with upstaging and upgrading in patients with low-risk prostate cancer who met the inclusion criteria for active surveillance. Urol Oncol 33: 201.e1-e8, 2015.

33. de Cobelli O, Buonerba C, Terracciano D, Bottero D, Lucarelli G, Bove $\mathrm{P}$, Altieri V, Coman I, Perdonà $\mathrm{S}$, Facchini $\mathrm{G}$, et al: Urotensin II receptor on preoperative biopsy is associated with upstaging and upgrading in prostate cancer. Future Oncol 11: 3091-3098, 2015

34. Sreekumar A, Poisson LM, Rajendiran TM, Khan AP, Cao Q, Yu J, Laxman B, Mehra R, Lonigro RJ, Li Y, et al: Metabolomic profiles delineate potential role for sarcosine in prostate cancer progression. Nature 457: 910-914, 2009. 